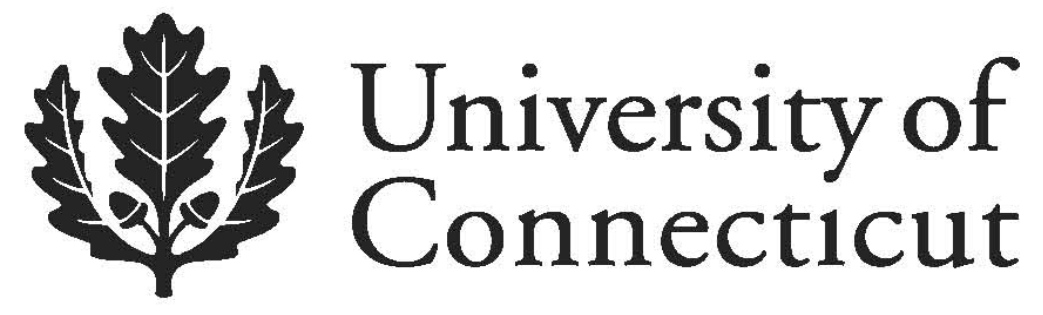

Department of Economics Working Paper Series

\title{
Designing a Sequential Choice Architecture to Reduce Choice Overload
}

Tibor Besedes

Georgia Institute of Technology

Cary Deck

University of Arkansas

Sudipta Sarangi

Louisiana State University, DIW Berlin

Mikhael Shor

University of Connecticut

Working Paper 2012-24

April 2012

365 Fairfield Way, Unit 1063

Storrs, CT 06269-1063

Phone: (860) 486-3022

Fax: (860) 486-4463

http://www.econ.uconn.edu/

This working paper is indexed on RePEc, http://repec.org 


\title{
Designing a Sequential Choice Architecture to Reduce Choice Overload*
}

\author{
Tibor Besedeš $\check{s}^{\dagger}$ \\ Georgia Institute of Technology \\ Sudipta Sarangi ${ }^{\S}$ \\ Louisiana State University \\ and DIW Berlin
}

\author{
Cary Deck ${ }^{*}$ \\ University of Arkansas \\ Mikhael Shor $^{* *}$ \\ University of Connecticut
}

April 2012

\begin{abstract}
Previous studies have demonstrated that a multitude of options can lead to choice overload, reducing decision quality. Through controlled experiments, we examine sequential choice architectures that enable the choice set to remain large while potentially reducing the effect of choice overload. A specific tournament-style architecture achieves this goal. An alternate architecture in which subjects compare each subset of options to the most preferred option encountered thus far fails to improve performance due to the status quo bias. Subject preferences over different choice architectures are negatively correlated with performance, suggesting that providing choice over architectures might reduce the quality of decisions.
\end{abstract}

\section{JEL Codes: C91, D03}

Key Words: choice architecture, choice overload, status quo bias, self-sorting, decision making, experiments

\footnotetext{
* This research was supported by the NIH National Institute on Aging grant R21AG030184.

${ }^{\dagger}$ School of Economics, Georgia Institute of Technology, Atlanta, GA 30332-0615, besedes@gatech.edu.

\$ 425 WCOB, Department of Economics, The University of Arkansas, Fayetteville, AR 72701, Fayetteville, AR 72701, cdeck@walton.uark.edu.

${ }^{\S}$ Department of Economics, 2107 Patrick Taylor Hall, Louisiana State University, Baton Rouge, LA 70803-6306, sarangi@1su.edu.

** Department of Economics, University of Connecticut, 341 Mansfield Road, Storrs, CT 06269-1063, mike.shor@uconn.edu.
} 


\section{Introduction}

Many decisions involve large choice sets from which one option must be selected. Financial retirement planning and health care insurance selection both present individuals with a seemingly limitless number of options. For example, Medicare participants can be confronted with over one hundred health insurance and prescription drug plans. Even less-consequential decisions often involve large choice sets, including shopping for a car, a cell phone plan, or a box of cereal. Traditional economic theory holds that more choice is better as the optimum over a proper subset can never be larger than the optimum over the original set. While a rational decision maker benefits from choice, studies have found that larger choice sets can reduce one's satisfaction with the decision (Iyengar and Lepper 2000), the likelihood of making a decision (Redelmeier and Shafir 1995, Iyengar and Lepper 2000, Roswarski and Murray 2006), and the quality and optimality of the decision (Payne et al. 1993, Tanius et al. 2009, Schram and Sonnemans 2011, Hanoch et al. 2011, and Besedeš et al. 2012).

Since decisions made from larger choice sets suffer from the above mentioned issues, one way of dealing with choice set complexity is simply to reduce its size. However, such an approach clearly has many undesirable attributes, chief among which are ethical concerns over paternalism and the reduction in some individuals' ability to obtain their most preferred option. Alternatively, one can ask what tools can assist decision makers while maintaining a plethora of options. Some have suggested a form of "libertarian paternalism" that nudges toward a decision while preserving all options (Sunstein and Thaler 2003), such as presenting additional options only if an individual requests them (Iyengar and Jiang 2000). The effectiveness of this approach relies on an assumption that people who request the additional options are benefitted by them, and those who do not are benefitted by the smaller choice set. We examine experimentally the ability of different choice architectures to improve decision making. Additionally, as people are likely heterogeneous in their decision-making approaches, we examine individuals' ability to identify their most suitable choice architecture. 
The choice architectures we consider reduce a large decision problem into a series of smaller ones. Such procedures approach a problem sequentially, eliminating a few options at a time. Sequential elimination techniques have been recommended for managerial decision making (Stroh et al. 2008) and patient counseling (Oostendorp et al. 2011), and are enshrined in the rules of parliamentary procedure (Robert et al. 2011). Nevertheless, to the best of our knowledge, their ability to stimulate optimal decision-making has not been considered.

The choice set we consider consists of lotteries structured in such a way that choices can be objectively ranked independent of personal idiosyncratic preferences. The benchmark simultaneous choice procedure involves picking one option among sixteen possibilities considered at once, a large enough number of options that choice overload has been found to be problematic (Tanius et al. 2009, Hanoch et al. 2011, Besedeš et al. 2012). Additionally, we consider two sequential procedures, with subjects considering subsets of the 16 options over several rounds. In the sequential elimination architecture, the decision maker first selects among four randomly-provided options. Then, the three options that were not selected are eliminated and replaced with three new options alongside the previously-selected one. This procedure repeats for a total of five rounds, until all 16 options have been considered. In the sequential tournament architecture, the 16 options are randomly divided into four sets of four options each. In the first four rounds, the decision maker selects one option from each of the four smaller sets. In the final round, the subject selects from among the four previously-selected options. Both sequential architectures involve subjects working through five rounds with four options in each but differ in whether the previously-selected option is carried into the next round (sequential elimination) or into a final round (sequential tournament).

After subjects make decisions under all three choice architectures, we elicit their preferences over the three choice architectures. The computer then randomly eliminates one of the three architectures and subjects complete another task in the more preferred of the two remaining architectures. This allows us to examine whether subject preferences coincide with the architecture that leads each to the best decision. 
A rational decision maker who evaluates the expected profit from each option and selects the optimal one from each choice set should not be affected by the simultaneous or sequential nature of the decision-problem. Conversely, a subject susceptible to choice overload may benefit from smaller choice sets. Regardless of the benefits of smaller choice sets, the introduction of sequential choice also changes decision making (Read and Loewenstein 1995). Sequential decisions are subject to inertia, or the status quo bias, by which the most recent selection is likely to be maintained (Agnew et al. 2003) in the next decision. While the sequential elimination architecture is perhaps more intuitive, its carryover of the selected option into the next decision round may exacerbate the status quo bias. The sequential tournament architecture in which previously-selected options appear together in the final round may mitigate this effect.

We identify three main results. First, we find that the sequential tournament generates the best overall performance. Second, sequential elimination offers no improvement over simultaneous choice due to the presence of significant inertia in subjects' sequential decisions. Third, while the sequential tournament generates the best performance, this choice architecture is least preferred by subjects of the three considered. We find evidence of adverse self-sorting, by which a portion of subjects select personally-suboptimal choice architectures. This suggests that allowing individuals to select their preferred choice architecture need not lead to improvements in decision making.

\section{Experimental Design and Procedures}

Subjects participated in a computerized experiment consisting of four decision tasks. Every decision task contained sixteen options and twelve potential states of nature. Options were characterized by a payment of $\$ 25$ under some states of nature, and zero otherwise. After each decision task, a state of nature was randomly drawn from a known probability distribution. If the option that the subject selected contained the drawn state of nature, the subject earned $\$ 25$ for that task. Thus, the optimal option was the option for which the prize was paid with the greatest 
probability. The key feature of this framework is that it allows for an objective evaluation and ranking of options independent of subjects' risk preferences (Besedeš et al. 2012).

While each task involved selecting one of sixteen options, the choice architecture, or process that governed the selection, varied. Three different choice architectures were employed. First, in the simultaneous choice procedure, subjects selected one option from all sixteen displayed at once. Figure 1 presents a sample screenshot of this task. To the subjects, the states of nature were presented as cards numbered 1 to 12 . The likelihood of a particular state was reflected in the frequency with which that card type appeared in a deck of 100 cards and presented in the "Odds" column. The sixteen options were labeled A through P and payment for certain cards (states) was denoted by a checkmark. In this example, the deck contains three Card 1s, five Card 2s, 4 Card 3s, and so on. A person who selected Option A would earn $\$ 25$ for this task if a Card 1, Card 3, Card 5, Card 7, Card 8, or Card 11 were randomly drawn and would receive zero otherwise. Thus, the likelihood of a Card 1 being drawn is 3 out of 100 and the likelihood that Option A results in payment is $3+4+9+4+12+15=47$ out of 100 .

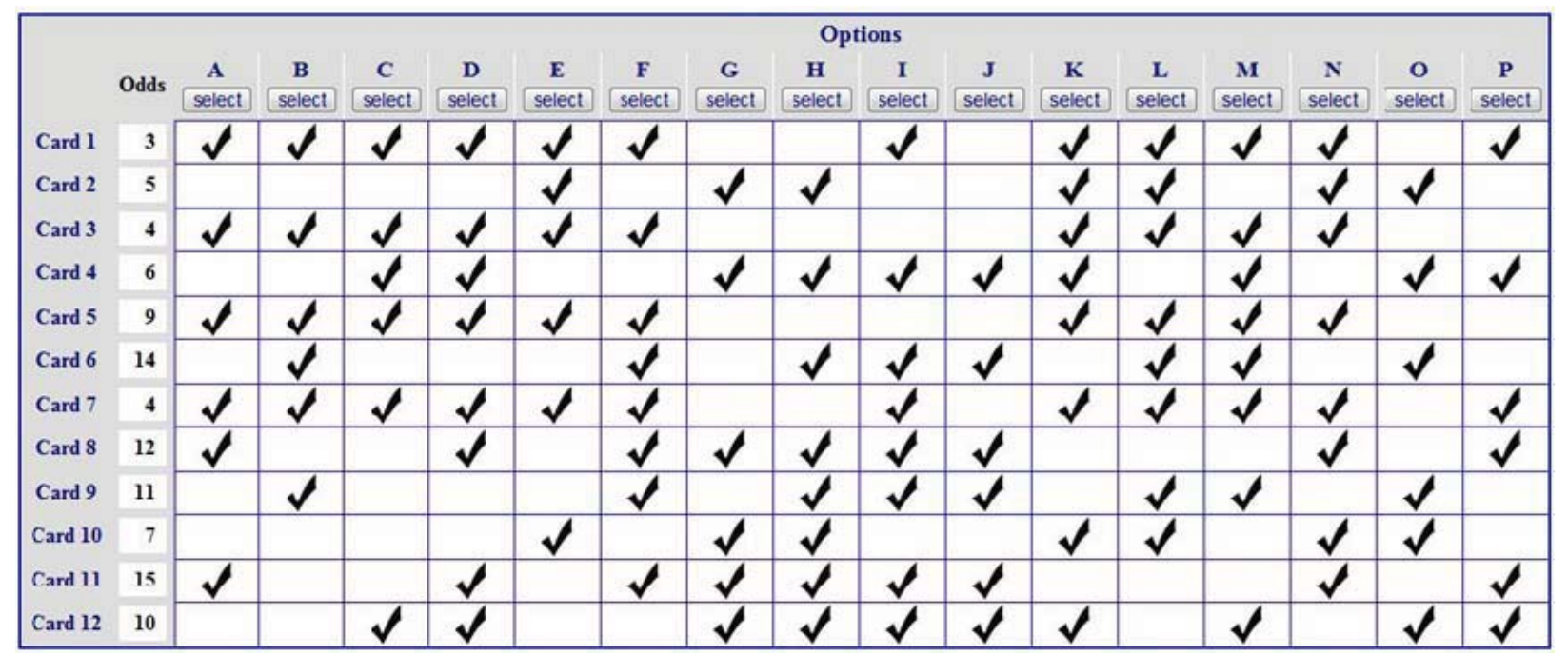

Figure 1: Screenshot of a Sample Simultaneous Choice Procedure

Second, in the sequential elimination architecture, subjects selected one option from sixteen possible options through a series of five rounds. In the first round, Options A through D were presented and one was selected. The selected option, along with Options E through G, was 
presented in round two. The option selected in round 2 was then presented along with Options $\mathrm{H}$ through $\mathrm{J}$ in round three, and so on through rounds four and five until all sixteen options had been presented. The final (fifth round) decision was the subject's selected option for the task.

Third, in the sequential tournament architecture, subjects also selected one option from sixteen possible options through a series of five rounds. In the first round, Options A through D were presented and one was selected. In the second round, Options $\mathrm{E}$ through $\mathrm{H}$ were presented and one was selected, and so on. By the end of the fourth round, the subject had seen all 16 options and selected one from each round. These four previously selected options were then presented in the fifth round and the final choice was made. The difference between sequential elimination and sequential tournament is that in sequential elimination the option selected in one round appears again in the next round, whereas in sequential tournament, a selected option does not reappear until the final round. ${ }^{1}$

Subjects were first required to complete a task using each of the three choice architectures, the order of which was randomized for each subject. Architecture-specific instructions were provided just before completing each task and subjects learned their earnings from each task at the end of that task. Prior to the fourth task, subjects provided a ranking of the three architectures which were used to select the choice architecture for the fourth task. This was incentivized by having the computer randomly eliminate one of the three choice architectures and implement the more preferred of the two remaining choice architectures for the fourth task. Subjects received instruction that it is in their best interest to reveal their preferences truthfully as the procedure yielded a $2 / 3$ chance of using the choice architecture reported as being most preferred and no chance of using the one reported as being least preferred. While subjects experienced the three choice architectures in a random order over the first three tasks, this preference procedure was always last so that subjects could make an informed decision.

To provide four similar, but not identical, choice tasks, the probability distributions were altered slightly across decision tasks. The four choice tasks are described in Table 1 . The four

\footnotetext{
${ }^{1}$ In experimental instructions we referred to simultaneous choice as select one, to sequential elimination as keep one, and to sequential tournament as send to final. These terms describe what subjects do in each task.
} 
probability distributions, PDF1 through PDF4, have similar probabilities for the most and least likely outcomes and nearly identical average probabilities across options (between 56.3 and 56.4). The black areas in Table 1 represent the states covered by each option. No two options are identical either in terms of the states contained, or in terms of expected value under any of the PDFs. The optimal option resulted in receiving the prize with approximately an $80 \%$ chance while the worst option yielded the prize with approximately $34 \%$ chance. To further ensure that the choice tasks appeared significantly different to subjects, the order of PDFs across tasks, and of options and states within tasks, were randomized. Thus, the subjects faced four similar decision problems, but could not use information about one problem on a subsequent one. Notably, subjects had the ability to provide post-experiment feedback, with none noting any similarity in the underlying set of options across tasks.

Table 1: Choice Tasks

\begin{tabular}{|c|c|c|c|c|c|c|c|c|c|c|c|c|c|c|c|c|c|c|c|c|}
\hline \multirow[t]{2}{*}{ Card } & \multicolumn{4}{|c|}{ PDF } & \multicolumn{16}{|c|}{ Options } \\
\hline & 1 & 2 & 3 & 4 & A & B & C & D & E & $\mathrm{F}$ & G & $\mathrm{H}$ & 1 & $\mathrm{~J}$ & K & $\mathrm{L}$ & M & $\mathrm{N}$ & 0 & $\mathrm{P}$ \\
\hline 1 & 15 & 13 & 13 & 12 & & & & & & & & & & & & & & & & \\
\hline 2 & 14 & 14 & 14 & 12 & & & & & & & & & & & & & & & & \\
\hline 3 & 12 & 11 & 12 & 9 & & & & & & & & & & & & & & & & \\
\hline 4 & 11 & 8 & 8 & 14 & & & & & & & & & & & & & & & & \\
\hline 5 & 10 & 12 & 10 & 11 & & & & & & & & & & & & & & & & \\
\hline 6 & 9 & 7 & 5 & 10 & & & & & & & & & & & & & & & & \\
\hline 7 & 7 & 6 & 9 & 5 & & & & & & & & & & & & & & & & \\
\hline 8 & 6 & 7 & 7 & 8 & & & & & & & & & & & & & & & & \\
\hline 9 & 5 & 9 & 6 & 7 & & & & & & & & & & & & & & & & \\
\hline 10 & 4 & 5 & 9 & 6 & & & & & & & & & & & & & & & & \\
\hline 11 & 4 & 4 & 4 & 3 & & & & & & & & & & & & & & & & \\
\hline \multirow[t]{7}{*}{12} & 3 & 4 & 3 & 3 & & & & & & & & & & & & & & & & \\
\hline & \multicolumn{4}{|c|}{ States Covered: } & 8 & 8 & 8 & 6 & 8 & 8 & 8 & 8 & 6 & 6 & 6 & 8 & 6 & 6 & 6 & 6 \\
\hline & \multicolumn{20}{|c|}{ Expected Payoffs: } \\
\hline & & & PDF & 1 & 80 & 75 & 72 & 68 & 63 & 61 & 59 & 57 & 55 & 53 & 50 & 48 & 47 & 45 & 36 & 32 \\
\hline & & & PDF & 2 & 80 & 73 & 66 & 65 & 63 & 61 & 59 & 57 & 58 & 56 & 51 & 54 & 44 & 42 & 39 & 35 \\
\hline & & & PDF & 3 & 79 & 71 & 68 & 64 & 63 & 60 & 61 & 58 & 57 & 54 & 49 & 53 & 46 & 43 & 38 & 36 \\
\hline & & & PDF & 4 & 78 & 72 & 69 & 66 & 62 & 67 & 55 & 60 & 52 & 57 & 46 & 53 & 43 & 48 & 41 & 34 \\
\hline
\end{tabular}


Prior to the four tasks of interest, subjects reviewed instructions and completed a fouroption four-state task to familiarize them with the computer interface. After selecting among the options in a task, subjects were shown a deck of cards reflecting the appropriate PDF. A subject then had the computer turn the cards face down and shuffle the deck, following which she chose one card. If the chosen card reflected a state covered by the selected option, the subject earned $\$ 25$ for the task. For example, if the subject selected option G in Figure 1 and then chose Card 2, she would earn $\$ 25$ for that task. However, if she chose Card 6 instead, the earnings for that task would be $\$ 0$.

After the experiment was completed, one of the four tasks was randomly selected for payment. The subject was paid his or her earnings for that task. In addition, the subjects were paid a $\$ 5$ participation fee. The average salient earnings were $\$ 17.66$ exclusive of the $\$ 5$ participation payment, while the average amount of time spent in the experiment was 26 minutes, of which five and a half minutes were spent on instructions and the rest on actively completing all tasks. The experiment was conducted through Vanderbilt University's eLab, an online lab with a pool of more than 70,000 subjects who have expressed a willingness to participate in experiments. Consistent with eLab policies, the subjects were mailed a check for their earnings in this study immediately after participating. eLab recruits subjects into its pool using links from partner sites, online advertisements, referrals from other panelists, and links from online search results, among other sources. eLab collects information on age, sex, and educational attainment from members of its subject pool, allowing us to capture this demographic information without collecting the information directly during the study. Our subject pool was $51 \%$ male with an average age of 48 years (standard deviation of 16). In terms of educational attainment, $30 \%$ of the subjects were college graduates, $32 \%$ had some college, and $38 \%$ had no schooling beyond high school. 


\section{Results}

\section{A. Choice Architecture and Quality of Decisions}

We begin with a summary of overall performance on the first three tasks using two different measures. The first measure, "Optimal Choice," is the frequency with which subjects select the option that yields the highest likelihood of payment. The second measure reflects how far the selected option is from the optimal one. It is equal to the difference between the probability of receiving payment under the optimal option and the probability of receiving payment under the selected option. We refer to this measure as "Money Left on the Table" since it reflects the reduction in the probability of payment from suboptimal choice. Across all tasks, subjects select optimally $28 \%$ of the time and selected options have an average probability of payment that is 14 percentage points lower than the optimal option. Thus, with $\$ 25$ at stake, subjects on average earn $\$ 3.50(=\$ 25 \times 0.14)$ less than they would with optimal choices. ${ }^{2}$

Results are presented in Table 2 . The sequential tournament leads to a significantly higher frequency of optimal choice than either simultaneous choice or sequential elimination (Wilcoxon $p=0.011$ and 0.029 , respectively), while sequential elimination and simultaneous choice are not significantly different $(p=0.470)$. Sequential tournament also leads to less money left on the table than simultaneous choice $(p=0.047)$, while sequential elimination is not significantly different from simultaneous choice $(p=0.864)$.

Table 2 - Average Performance across Choice Architectures

\begin{tabular}{lcc}
\hline \hline & Optimal Choice & Money Left on the Table \\
\cline { 2 - 3 } Simultaneous choice & $23 \%$ & 0.14 \\
Sequential elimination & $25 \%$ & 0.14 \\
Sequential tournament & $36 \%$ & 0.12 \\
\hline \hline
\end{tabular}

To control for confounding effects of task and demographic characteristics, we estimate a probit regression for optimal choice and an OLS regression for money left on the table. We have

\footnotetext{
${ }^{2}$ The main results are qualitatively unchanged if we use alternative measures of efficiency, such as the ratio of payoffs of the chosen and optimal options.
} 
a total of 333 observations, 3 for each subject. We include demographic variables for age, sex, and dummies for educational attainment (some college and college graduate, with high school the omitted variable). We also include PDF and task order fixed effects (suppressed for brevity). Treatment dummy variables for the sequential choice architectures are included (with simultaneous choice as the omitted variable).

Table 3 - Factors Influencing Choice Quality

\begin{tabular}{lcc}
\hline \hline & Optimal Choice & Money Left on the Table \\
\cline { 2 - 3 } Sequential elimination & 0.108 & -0.443 \\
Sequential tournament & $(0.128)$ & $(1.368)$ \\
& $0.448^{* * *}$ & $-2.164^{*}$ \\
Age & $(0.163)$ & $(1.151)$ \\
& -0.006 & 0.061 \\
Male dummy & $(0.006)$ & $(0.049)$ \\
& -0.087 & 2.167 \\
Some college & $(0.202)$ & $(1.690)$ \\
& $0.678 * * *$ & $-5.608 * * *$ \\
College graduate & $(0.240)$ & $(2.059)$ \\
& $0.584 * *$ & $-5.892^{* * *}$ \\
Constant & $(0.253)$ & $(1.890)$ \\
& $-0.670 *$ & $12.141^{* * *}$ \\
Observations & $(0.392)$ & $(3.275)$ \\
\hline log likelihood & 333 & 333 \\
\hline \hline Probit coefficients reported for optimal choice, OLS for Money Left on the Table. Robust standard errors in \\
parentheses, with ***,**, and * denoting significance at the 1\%, 5\%, and 10\% levels. PDF and task order \\
fixed effects included, but not reported. Money left on the table was measured on a 0-100 scale.
\end{tabular}

The regression results in Table 3 confirm the relative performance in Table 2. Sequential elimination does not lead to significant improvement over simultaneous choice, while the sequential tournament architecture significantly improves the quality of choices, both in terms of increased frequency of optimal choice and reduced amount of money left on the table. Of the demographic variables, age and sex appear to play no role while education beyond high school is correlated with an estimated twenty percentage point increase in the chance of selecting the optimal option. Averaging across subjects, the sequential tournament architecture leads to a 
predicted fourteen percentage point increase in optimal choice frequency relative to simultaneous choice. Conversely, sequential elimination leads to no significant improvement.

\section{B. Choice Overload and the Status Quo Bias}

The choice overload hypothesis suggests that smaller choice sets can result in better decisions. In our experiment, each round in the sequential elimination and sequential tournament architectures involves a choice among only four options whereas the simultaneous decision architecture involves a choice among sixteen options. We first examine whether decision making is better in 4-option choices than in 16-option choices (Table 4). Here, measures of optimal choice and money left on the table are relative to the set of options in each round. Thus, for the simultaneous decision, these measures coincide with those in Table 3, but do not for the other two architectures. We again consider only the first three tasks, and exclude the fourth task in which subjects chose the choice architecture. The frequency of optimal choice when selecting among 16 options at once is only $23 \%$, while the average across all rounds in sequential architectures is $47 \%$. This is consistent with choice overload.

Table 4 - Round-by-Round Performance

\begin{tabular}{lcc}
\hline \hline Task & Optimal Choice & Money Left on the Table \\
\cline { 2 - 3 } Simultaneous choice (16 options) & $23 \%$ & 0.14 \\
Sequential elimination (4 options) & $46 \%$ & 0.08 \\
Sequential tournament (4 options) & $48 \%$ & 0.08 \\
\hline \hline
\end{tabular}

Our results so far indicate decision making is better when fewer options are considered at once, but that the way a large set of options is broken into smaller parts matters for the quality of the final decision. Given this result, we try to understand why performance in the sequential tournament architecture is superior to performance in the sequential elimination architecture even though both entail the same number of decisions over choice sets of the same size. We offer two possible explanations.

First, we consider the possibility that subjects simply make independent errors in each round. These independent errors, even if equal across architectures, produce different rates of 
optimal choice among all sixteen options for the two sequential architectures. For a subject to select the optimal option in the sequential tournament architecture, she must select optimally in two rounds: the round in which the option first appears, and the final round. For the sequential elimination architecture, the subject must select optimally in the first round in which the optimal option appears, and in each subsequent round. Statistically, this makes the chance of selecting the optimal option higher in the sequential tournament architecture than in the sequential elimination architecture. ${ }^{3}$ In our case, the $46 \%$ chance of selecting the best option in each round of sequential elimination would translate into a $16 \%$ of selecting the optimal option overall under the assumption of independent errors. For the sequential tournament architecture, the $48 \%$ in each round translates into a $23 \%$ of selecting the optimal option in the final round. The actual rates from Table 2 are substantially higher for both architectures, suggesting that simple independent error rates cannot fully explain our results.

Second, we consider the possibility that errors are not independent across rounds due to the status quo bias. The selection of an option in one round may cause a subject to overvalue that same option in the next round, or view selecting another option as a psychologically costly disaffirmation of their previous choice (Kahneman et al. 1991). Sequential elimination may lay a trap for subjects susceptible to the status quo bias by carrying a selected option over to the next round. An error in selection in one round is likely to persist as the subject continues to select the same option in subsequent rounds. Conversely, in the sequential tournament architecture, all options presented concurrently are on equal footing: either none has been previously considered or, in the final round, all have been selected in a previous round.

We use McFadden's (1974) conditional logit model to estimate subject choices in each round as a function of two predictive variables: (i) the expected payoff, or expected probability of payment of each option, which proxies for optimal choice, and (ii) in the sequential

\footnotetext{
${ }^{3}$ Denote by $p$ the probability of selecting the best option in each round. For the sequential tournament architecture, this translates into a probability of ultimately selecting the optimal option of $p^{2}$. For the sequential elimination architecture, the probability that the optimal option appears in the first round is $4 / 16$, and it is $3 / 16$ for subsequent rounds. Thus, the probability of selecting the optimal option is $\frac{3}{16} p\left(1+p+p^{2}+p^{3}+\frac{4}{3} p^{4}\right)$. The sequential tournament architecture leads to a higher probability of selecting the optimal option whenever $p>1 / 4$.
} 
elimination architecture, whether the option was selected in the previous round. Specifically, "Expected Payoff" is coded as the probability of payment, between zero and one. The "Selected Previous Round" dummy equals one for the options in rounds two through five of the sequential elimination architecture that were selected in the previous round, and equals zero for all other options. We consider three subsets of data: decisions in each of the sequential architectures separately and pooled. Table 5 presents the estimates.

Table 5 - Status Quo Bias

\begin{tabular}{lcccc}
\hline \hline \multirow{2}{*}{ Expected Payoff } & Sequential Elimination & Sequential Tournament & & Pooled Sequential \\
\cline { 2 - 2 } Selected Previous Round & $4.627^{* * *}$ & $5.324^{* * *}$ & $4.925^{* * *}$ \\
& $(0.631)$ & $(0.601)$ & $(0.513)$ \\
& $0.459^{* * *}$ & & $0.550^{* * *}$ \\
Observations (Options) & $(0.134)$ & & $(0.125)$ \\
Observations (Decisions) & 2,220 & 2,220 & 4,440 \\
log likelihood & 111 & 111 & 222 \\
\hline \hline
\end{tabular}

The significance of expected payoff indicates that better options are selected with higher probability. The significance of selected previous round suggests that subjects exhibit a status quo bias in the sequential elimination tasks. Given the within-subject nature of our design, the consistency of the payoff heuristic across architectures is not surprising. ${ }^{4}$ Yet, when the status quo bias is provided an opportunity to manifest, subjects change their decision-making approach to place additional reliance on the option previously selected. The relative parameter magnitudes indicate that the status quo bias is equivalent to approximately $10(=4.627 / 0.459)$ percentage points of the probability of payment. For example, a previously-selected option with a $70 \%$ chance of payment has a similar probability of being selected as a new option with an $80 \%$ chance of payment. Thus, status quo bias causes subjects to stick with options they selected, even if they are not optimal.

\footnotetext{
${ }^{4}$ We pool across both sequential architectures to show the consistency of the payoff variable. By the nature of the conditional logit estimator, separate regressions do not allow direct comparisons of parameters due to their confluence with potentially different variances. Confidence that these are similar is gained in column three which imposes identical variance on both.
} 


\section{Preferences for Choice Architecture}

Thus far, our results have focused on how the choice architecture impacts decision quality. We now examine which choice architecture subjects prefer, and how those preferences correlate with the quality of their decisions. More than a half of our subjects, 59 out of 111, preferred the simultaneous choice architecture, while 29 (26\%) preferred sequential elimination, and the remaining 23 (21\%) preferred the sequential tournament. These preferences run opposite to the proportion of subjects selecting optimally under each architecture. The joint preference ranking of the least preferred choice architecture is almost a mirror image of the most preferred ranking. Just over a half of our subjects, 56, revealed sequential tournament as the least preferred architecture, followed by $30(27 \%)$ who rated sequential elimination as the least preferred, and $25(23 \%)$ who rated simultaneous choice as the least preferred architecture.

Given our procedure for eliciting rankings, subjects had a 2/3 chance of using their most preferred architecture for the fourth task and a 1/3 chance of using their second-most preferred architecture. Table 6 reports overall performance on the fourth task by choice architecture, and includes performance from the first three tasks in parentheses for comparison. Again, performance is best under the sequential tournament architecture despite the fact that it is the least preferred. Table 6 also reveals a suggestive pattern. While performance in both sequential architectures is better the second time it is used (in task 4), performance in the simultaneous decision is actually worse the second time it is used than when it was first encountered. This suggests an adverse self-sorting in subjects' preferences for the simultaneous choice architecture.

Table 6 - Performance on Task 4 (Average Performance on Tasks 1-3)

\begin{tabular}{lcc}
\hline \hline Task & Optimal Choice & Money Left on the Table \\
\cline { 2 - 3 } Simultaneous choice $(\mathrm{N}=43)$ & $15 \%(23 \%)$ & $0.15(0.14)$ \\
Sequential elimination $(\mathrm{N}=33)$ & $27 \%(25 \%)$ & $0.11(0.14)$ \\
Sequential tournament $(\mathrm{N}=25)$ & $40 \%(36 \%)$ & $0.08(0.12)$ \\
\hline \hline
\end{tabular}

To explore the possibility of adverse self-sorting, we investigate the frequency with which subjects prefer the choice architectures on which they performed best initially. We focus on subjects whose performance under one architecture was strictly better than under the other 
two. For this purpose, we say a subject performed unambiguously best in a particular choice architecture if the rank of the selected option is higher in that architecture than in the other two. ${ }^{5}$ If a subject did equally well under two procedures then no unambiguously best architecture is identified. The data are tabulated in Table 7.

Table 7 - Unambiguously Best Architecture Performance and Architecture Preferences

\begin{tabular}{lccc}
\hline \hline & \multicolumn{3}{c}{ Most preferred architecture } \\
\cline { 2 - 4 } Unambiguously Best Architecture & $\begin{array}{c}\text { Simultaneous } \\
\text { choice }\end{array}$ & $\begin{array}{c}\text { Sequential } \\
\text { elimination }\end{array}$ & $\begin{array}{c}\text { Sequential } \\
\text { tournament }\end{array}$ \\
\hline Simultaneous choice $(\mathrm{N}=21)$ & $62 \%$ & $14 \%$ & $24 \%$ \\
Sequential elimination $(\mathrm{N}=22)$ & $36 \%$ & $50 \%$ & $14 \%$ \\
Sequential tournament $(\mathrm{N}=30)$ & $73 \%$ & $10 \%$ & $17 \%$ \\
\hline None $(\mathrm{N}=38)$ & $42 \%$ & $32 \%$ & $26 \%$ \\
\hline \hline
\end{tabular}

If subjects' preferences over choice architecture were associated with how well they performed in each, entries should fall along the diagonal in Table 7. Twenty-one subjects did best in simultaneous choice, of which $62 \%$ identified it as their most preferred architecture. Of the 22 subjects who did best in sequential elimination, 50\% identified it as their most preferred procedure. The most unexpected results are for those who do best in the sequential tournament. Of the 30 subjects who did best in sequential tournament, only $17 \%$ identified it as their most preferred architecture while $73 \%$ preferred simultaneous choice. This means that individuals who perform best in sequential tournament are more likely to prefer simultaneous choice than those who actually performed best in simultaneous choice. The 38 subjects for whom no unambiguously best architecture is identified exhibit a similar adverse self-sorting. While a plurality of these subjects prefer simultaneous choice, $85 \%$ do at least as well under the sequential tournament architecture.

To understand the source of subjects' preferences over architectures, we estimate a multinomial logit regression. The dependent variable is the preferred architecture. Our

\footnotetext{
${ }^{5}$ We obtain the same qualitative results if we consider only subjects who chose optimally under exactly one mechanism or by defining "unambiguously best" based on which architecture yielded the highest expected payoff or lowest amount of money left on the table. The challenge with the latter two definitions is that ordinally-equivalent choices lead to different payoffs due to slight variations across PDFs by design.
} 
independent variables are: expected payoff from the option chosen under each architecture, actual earnings under each architecture, total decision time of each sequential architecture relative to the simultaneous choice architecture, and demographic variables for age, sex, and education. The expected payoff from each option examines whether preferences are correlated with performance, while earnings examine whether subjects respond to actual (randomly determined) payoffs instead of those that are a priori optimal. The relative time measures examine if subjects aim to minimize decision time. On average, subjects spent $120 \%$ longer on sequential elimination and $90 \%$ longer on sequential tournament than on simultaneous choice. Results are presented in Table 8. Coefficients are presented as relative risk ratios for preferring each sequential architecture relative to simultaneous choice. Thus, coefficients less than one imply that the parameter makes preferring the sequential architecture less likely than simultaneous choice.

We find that the preference for sequential elimination is increasing with performance and decreasing with time. Specifically, a subject who takes twice as long on sequential elimination as simultaneous choice is $37 \%$ less likely to prefer sequential elimination to simultaneous choice as a subject who spends equal time on both. However, this extra time is exactly offset if sequential elimination leads to the selection of an option that is six percentage points better (in expected payoff) than the one selected under simultaneous choice. Additionally, we find that college graduates are substantially more likely to prefer simultaneous choice to sequential elimination beyond any performance-based or time-based reasons. We find no evidence that architecture preference is related to the probabilistic outcome of whether or not one actually earned a payment. For the sequential tournament architecture, we find no significant relationships between performance, time, and preference. The only significant variable is a curious spillover effect between performance in sequential elimination and preference for sequential tournament.

Overall, the analysis suggests that subjects quite deliberately consider the simultaneous choice and sequential elimination architectures, taking into account both decision time and decision performance, and on aggregate selecting the better of these two architectures. Unfortunately, by not considering as heavily the sequential tournament architecture, subjects are 
effectively deciding between two architectures that are both similar in aggregate performance and inferior to the least preferred architecture.

Table 8 - Determinants of Architecture Preferences

\begin{tabular}{|c|c|c|}
\hline & Elimination & Tournament \\
\hline \multicolumn{3}{|l|}{ Expected Payoff in ... } \\
\hline \multirow[t]{2}{*}{ Simultaneous choice } & 1.012 & 1.044 \\
\hline & $(0.029)$ & $(0.035)$ \\
\hline \multirow[t]{2}{*}{ Sequential elimination } & $1.060 * *$ & $1.050 * *$ \\
\hline & $(0.031)$ & $(0.024)$ \\
\hline \multirow[t]{2}{*}{ Sequential tournament } & $0.941 * *$ & 0.960 \\
\hline & $(0.025)$ & $(0.027)$ \\
\hline \multicolumn{3}{|l|}{ Actual Earnings in ... } \\
\hline \multirow[t]{2}{*}{ Simultaneous choice } & 1.018 & 0.502 \\
\hline & (0.619) & $(0.304)$ \\
\hline \multirow[t]{2}{*}{ Sequential elimination } & 0.765 & 0.706 \\
\hline & $(0.405)$ & $(0.431)$ \\
\hline \multirow[t]{2}{*}{ Sequential tournament } & 0.585 & 0.421 \\
\hline & $(0.350)$ & $(0.269)$ \\
\hline \multicolumn{3}{|l|}{ Relative decision time } \\
\hline Sequential elimination & $0.632 * *$ & 0.924 \\
\hline / simultaneous elimination & $(0.116)$ & $(0.178)$ \\
\hline Sequential tournament & 0.976 & 0.896 \\
\hline / simultaneous elimination & $(0.129)$ & $(0.127)$ \\
\hline \multirow[t]{2}{*}{ Age } & 1.009 & 1.019 \\
\hline & $(0.016)$ & $(0.018)$ \\
\hline \multirow[t]{2}{*}{ Male dummy } & 1.089 & 1.256 \\
\hline & $(0.655)$ & $(0.742)$ \\
\hline \multirow[t]{2}{*}{ Some college dummy } & 1.970 & 2.312 \\
\hline & (1.338) & $(1.549)$ \\
\hline \multirow[t]{2}{*}{ College graduates dummy } & $0.252^{*}$ & 1.294 \\
\hline & $(0.178)$ & $(0.828)$ \\
\hline \multirow[t]{2}{*}{ Constant } & 0.934 & 0.019 \\
\hline & $(2.304)$ & $(0.049)$ \\
\hline Observations & \multicolumn{2}{|c|}{111} \\
\hline log likelihood & \multicolumn{2}{|c|}{-95} \\
\hline
\end{tabular}




\section{Conclusions}

By now, several studies have suggested that increased choice may not be beneficial to decision makers. Despite the greater likelihood of a better option being available, a wider variety of choices may lead to choice overload, greater regret, and more indecision. This has led some to suggest that choice sets should be restricted (Schwartz 2005). From a practical standpoint, all proposals calling for restricting a choice set face the criticism of being paternalistic in determining how choices are restricted.

Instead of attempting to restrict the choice set, we seek to identify a choice architecture that will enhance decision quality while maintaining the size of the choice set. Consistent with previous work, we find that decision making improves when fewer options are considered concurrently. Thus, our focus is on two sequential processes that break a decision into a series of choices each among a small number of options. The intuitive and commonly suggested sequential elimination approach is actually not beneficial. When a previously-selected option is compared to a new subset of options, subjects exhibit a status quo bias which causes them to undervalue new options.

Our sequential tournament process does succeed in improving the quality of decision making. This choice architecture first places options into subgroups and then the options selected from each subgroup are combined into a final set from which the ultimate decision is made. It captures the advantage of a small choice set for each decision while avoiding the status quo bias.

While we find that a sequential tournament improves aggregate decision making, individuals prefer to make decisions from all of the options at once. In the aggregate, there is a negative correlation between architecture performance and architecture choice. We find evidence of adverse self-sorting with subjects preferring choice architectures in which they did not have their best performance. Thus, simply letting people select a choice architecture may be insufficient to facilitate improved decision making. 
Thaler and Sunstein (2008) argue for "libertarian paternalism," a decision-making intervention in which choice architectures direct towards certain choices while maintaining the opportunity to select among the full range of options. For example, Iyengar and Jiang (2000) suggest that people should initially be presented with only a few options while retaining the ability to consider a larger set of options if they so choose. The desirability of such a choice architecture inherently assumes that adverse self-sorting is not a problem and that only the right people expand the choice set. Specifically, for such an architecture to improve choice quality, preferences over choice set size and performance under different choice set sizes need to correspond.

Our findings essentially push the paternalistic discussion associated with choice overload back one level. More, but not all, people would make better decisions with a sequential tournament; however, this choice architecture is the least preferred of those we consider. Therefore, in some cases, policy makers or others designing a choice problem may wish to impose an unpopular procedure in order to improve decision making quality. Clearly, the appropriateness of such libertarian paternalism needs to be evaluated on a case-by-case basis. 


\section{References}

Agnew, Julie, Pierluigi Balduzzi, and Sunden Annika (2003), "Portfolio choice and trading in a large 401(k) plan," American Economic Review, 93(1): 193-215.

Besedeš, Tibor, Cary Deck, Sudipta Sarangi, and Mikhael Shor (2012), "Age Effects and Heuristics in Decision Making," Review of Economics and Statistics, 94(2), forthcoming.

Hanoch, Yaniv, Stacey Wood, Andrew Barnes, Pi-Ju Liu, and Thomas Rice (2011), "Choosing the right Medicare prescription drug plan: The Effect of Age, Strategy Selection and Choice Set Size," Health Psychology, 30(6): 719-727.

Iyengar, Sheena S., and Mark R. Lepper (2000), "When Choice is Demotivating: Can One Desire Too Much of a Good Thing?" Journal of Personality and Social Psychology, 79(6): 995-1006.

Iyengar, Sheena S., and Wei Jiang (2000), "The Psychological Costs of Ever Increasing Choice: A Fallback to the Sure Bet," Columbia University, mimeo.

Kahneman, Daniel, Jack L. Knetsch, and Richard H. Thaler (1991), “Anomalies: The Endowment Effect, Loss Aversion, and Status Quo Bias," Journal of Economic Perspectives, 5(1): 193-206.

McFadden, Daniel (1974), "Conditional Logit Analyses of Qualitative Choice Behavior,” in Paul Zarembka (ed.), Frontiers of Econometrics, pp. 105-142.

Oostendorp, Linda J.M., Petronella B. Ottevanger, Winette T.A. van der Graaf, and Peep F.M. Stalmeier (2011), "The Benefits of Discussing Adjuvant Therapies One at a Time instead of All at Once," Breast Cancer Research and Treatment, 129(1): 79-87.

Payne, John W., James R. Bettman, and Eric J. Johnson (1993), The Adaptive Decision Maker, Cambridge University Press, Cambridge, UK.

Read, Daniel, and George Loewenstein (1995), "The Diversification Bias: Explaining the Discrepancy in Variety Seeking between Combined and Separated Choices," Journal of Experimental Psychology: Applied, 1(1): 34-49.

Redelmeier, Donald A., and Eldar Shafir (1995), "Medical Decision Making in Situations that Offer Multiple Alternatives," Journal of the American Medical Association, 273(4): 302 305.

Robert, Henry M. III, Daniel H. Honemann, and Thomas J. Balch (2011), Robert's Rules of Order, 11th ed., De Capo Press, Philadelphia, PA.

Roswarski, Todd Eric, and Michael D. Murray (2006), "Supervision of Students May Protect Academic Physicians from Cognitive Bias: A Study of Decision-Making and Multiple Treatment Alternatives in Medicine," Medical Decision Making, 26(2): 154-161. 
Schram, Arthur, and Joep Sonnemans (2011), "How Individuals Choose Health Insurance: An Experimental Analysis," European Economic Review, 55(6): 799-819.

Schwartz, Barry (2005), The Paradox of Choice: Why More is Less, Harper Collins, New York, NY.

Stroh, Linda K., Gregory B. Northcraft, and Margaret A. Neele (2008), Organizational Behavior: A Management Challenge, 3rd ed., Lawrence Erlbaum Associates, Mahwah, NJ.

Sunstein, Cass, and Richard H. Thaler (2003), "Libertarian Paternalism," American Economic Review, 93(2): 175-179.

Tanius, Betty E., Stacey Wood, Yaniv Hanoch, and Thomas Rice (2009), “Aging and Choice: Applications to Medicare Part D," Judgement and Decision Making, 4(1): 92-101.

Thaler, Richard, and Cass Sunstein (2008), Nudge: Improving Decisions About Health, Wealth, and Happiness, Yale University Press, New Haven, CT. 


\title{
Appendix: Experiment Instructions
}

\section{General Instructions}

\author{
INTRODUCTION
}

You are participating in an experiment on decision making. You will receive $\$ 5$ for completing the experiment. At the end of the experiment, you may also be paid an additional $\$ 25$ based on your decisions during the experiment. It is important that you understand the instructions well since this can help you make better decisions and hence earn more money.

In this experiment you will complete four tasks. At the end of the experiment, one and only one of these four tasks will be randomly selected and your payment will depend on the choices you made for that task.

In each task, you will select one option from 16 possible options labelled $A, B, C, D, E, F, G, H_{2}, J_{2}, K_{2}, M_{2}, M_{2}, O, O$, and $P$. To make these instructions simple, the example to the right has only three options: $A$, $\mathrm{B}$, and $\mathrm{C}$. In each task, one card will be drawn from a deck of 100 cards. If the option you selected includes the card drawn, then you will earn an additional $\$ 25$ if the task is the one selected for payment. If the option does not include the card drawn, then you will not earn any additional money if the task is the one selected for payment.

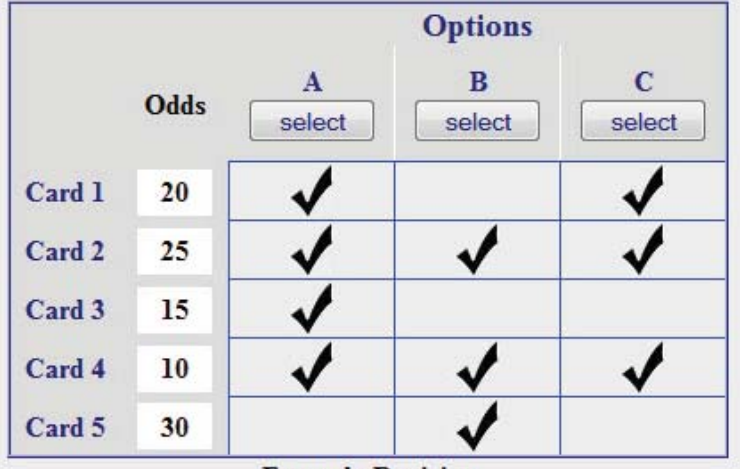

Example Decision

In this example, the cards are numbered from 1 through 5 and the numbers next to the cards in the column labelled "Odds" tell you how many cards in the deck are of that type. In the example, there are twenty cards in the deck with a 1, twenty-five cards with a 2 , and so on. This is also shown in the deck of cards below. Since there are 100 cards in total, the chance that a Card 1 is drawn is $20 / 100=20 \%$. In the four tasks in the experiment, there will be twelve cards numbered 1 through 12 . There will always be a total of 100 cards in all tasks, but the number of cards of each type will change from task to task. Since there are always 100 cards, the "Odds" column always gives you the chance that a card of a particular type will be drawn.

A check mark in the column under an option indicates that the option contains that particular kind of card. In the example, Option A has cards $1,2,3$, and 4, while Option B has cards 2,4 and 5. No two options will have the same set of cards.

You select an option by clicking the "select" button corresponding to the option you want. When you press the "select" button, a dialog box will open asking you to confirm your choice. You may try this now by pressing the "select" button for an option in the table.

The specific way that you select one option from among the sixteen available options will differ from task to task. Instructions for each task will explain how you select an option in that particular task. After you select an option, a card will be drawn. Suppose that a Card 3 was drawn. You would earn $\$ 25$ for the task if you had selected option A, but would not earn any money if you had selected Option B or Option C. This is because Option A has a check mark for Card 3 but Option B and Option C do not. We will now describe in detail how the process of drawing a card works. 
In each task, after you select an option, you will have to draw one card from the deck of cards. Each task will have a deck of 100 cards and the "Odds" column will tell you how many cards of each type are in the deck. To the right is a sample deck of 100 cards corresponding to the example decision shown above. Recall there are 20 Card 1's, 25 Card 2's, and so on.

Before selecting a card, you will have to shuffle the cards. By clicking on the "Shuffle" button, the cards will be turned over and shuffled. If you have not already done so, you should press the "Shuffle" button now to see how this works.

After shuffling ends, you can click on any one card to select it. Clicking on a card will reveal the card you have drawn. If you have not already done so, you should select a card now by clicking on it. If your selected option contains the card you selected you will earn $\$ 25$ for that task. If your selected option does not contain the card you chose, then you will receive no additional earnings for that task. During the process of shuffling and choosing cards you will be able to see your selected option below the deck of cards.

Once you choose a card from the shuffled deck, you will have to reveal all cards in the deck by clicking the "Reveal All Cards" button. If you have not already done so, you may press on the "Reveal All Cards" button now.

At this point, your results for the task would be displayed. These results will tell you (1) whether your selected option contains the card you drew, (2) your earnings for the current task, and (3) your earnings in each previously completed task. You will then be able to press a button to proceed to the next round.

\section{When instructed,}

press the button to shuffle the cards:

\section{Shuffle}

\begin{tabular}{|l||l|l|l||l|l|l|l|l|l|}
\hline 1 & 1 & 1 & 1 & 1 & 1 & 1 & 1 & 1 & 1 \\
\hline \hline 1 & 1 & 1 & 1 & 1 & 1 & 1 & 1 & 1 & 1 \\
\hline 2 & 2 & 2 & 2 & 2 & 2 & 2 & 2 & 2 & 2 \\
\hline 2 & 2 & 2 & 2 & 2 & 2 & 2 & 2 & 2 & 2 \\
\hline 2 & 2 & 2 & 2 & 2 & 3 & 3 & 3 & 3 & 3 \\
\hline 3 & 3 & 3 & 3 & 3 & 3 & 3 & 3 & 3 & 3 \\
\hline 4 & 4 & 4 & 4 & 4 & 4 & 4 & 4 & 4 & 4 \\
\hline 5 & 5 & 5 & 5 & 5 & 5 & 5 & 5 & 5 & 5 \\
\hline \hline 5 & 5 & 5 & 5 & 5 & 5 & 5 & 5 & 5 & 5 \\
\hline \hline 5 & 5 & 5 & 5 & 5 & 5 & 5 & 5 & 5 & 5 \\
\hline
\end{tabular}

The Deck of Cards 


\section{SUMMARY}

To sum up, this experiment consists of 4 tasks. In each task, you will be presented with 16 options, each containing different combinations of 12 cards. The numbers next to the cards denote how many of each card type are in the deck of 100 cards. After you pick an option, the computer shuffles the deck and you draw one card. If you select an option that contains the drawn card, you will earn $\$ 25$ for that task. After you have completed all four tasks, one will be randomly chosen and you will be paid based upon your earnings for that task, in addition to your \$5 participation payment.

Before starting your four tasks, you will first have a practice task which will demonstrate how options are selected, how a card is drawn, and how your earnings are determined. This practice task has no impact on your earnings today.

Please note that each task is a separate decision-making problem. You can only select one option in any task, but you can select different options in different tasks. At no time during this experiment will you be able to return and change your decision.

\section{READY?}

To move through the experiment, you should use only the buttons provided. Do not use any of your browser's buttons ("Forward," "Back," or "Refresh") as this will void the experiment and the payment you have earned.

When you have read the instructions and are ready to proceed, press the button below.

Proceed to Experiment 


\section{Practice Decision Task}

-Instructions

This is a practice task and will have no impact on your payment today.

In this task, you will select one option from 4 possible options: A, B, C, and D. You select an option by clicking the "select" button corresponding to the option you want. When you press the "select" button, a dialog box will open asking you to confirm your choice. You may try selecting an option now by pressing the "select" button for an option in the table.

Please select an option from the table below. We will then go through the process of determining your earnings using a deck of cards as explained in the instructions even though this task will not have any impact on your payment. The deck of cards that applies to this decision is shown below the table of options.

-Table Layout

If you want to change how the table below looks on your screen, you can change the layout by clicking on one of the images below. Please note that this will not change the information in the table in any way.

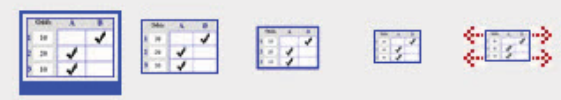

Practice Task

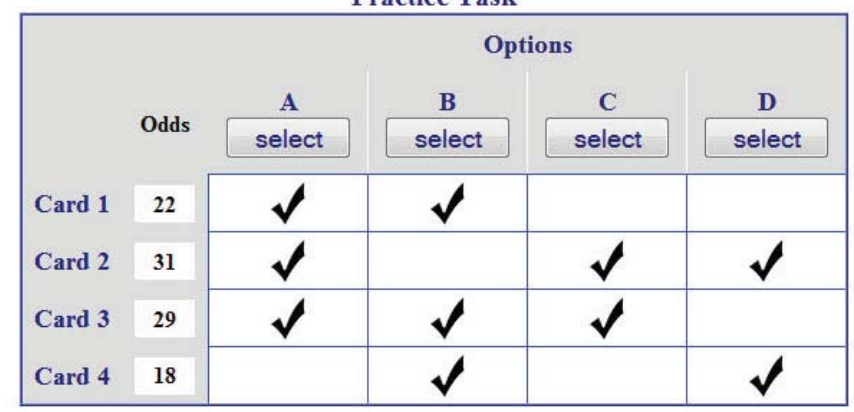

\begin{tabular}{|l|l|l|l|l|l|l|l|l|l|}
\hline 1 & 1 & 1 & 1 & 1 & 1 & 1 & 1 & 1 & 1 \\
\hline \hline 1 & 1 & 1 & 1 & 1 & 1 & 1 & 1 & 1 & 1 \\
\hline \hline 1 & 1 & 2 & 2 & 2 & 2 & 2 & 2 & 2 & 2 \\
\hline 2 & 2 & 2 & 2 & 2 & 2 & 2 & 2 & 2 & 2 \\
\hline \hline 2 & 2 & 2 & 2 & 2 & 2 & 2 & 2 & 2 & 2 \\
\hline \hline 2 & 2 & 2 & 3 & 3 & 3 & 3 & 3 & 3 & 3 \\
\hline 3 & 3 & 3 & 3 & 3 & 3 & 3 & 3 & 3 & 3 \\
\hline \hline 3 & 3 & 3 & 3 & 3 & 3 & 3 & 3 & 3 & 3 \\
\hline 3 & 3 & 4 & 4 & 4 & 4 & 4 & 4 & 4 & 4 \\
\hline 4 & 4 & 4 & 4 & 4 & 4 & 4 & 4 & 4 & 4 \\
\hline
\end{tabular}




\section{Decision Task - Send to Final}

-Instructions

In this task, you will select one option from 16 possible options: $A, B, C, D, E, F, G_{2}, H_{2}, J_{2}, L_{2}, M_{2} N, O_{2}$, and $P$. This choice is made in a series of five rounds.

In the first round, you will be shown 4 options: A, B, C and D. You will select one of these four options to go to the final round. In the second round, you will be shown 4 new options: E, F, G, and H. Again, you will select one of these four options to go to the final round. This process will continue for two more rounds with new options. Then, in the fifth and final round, you will be shown the four options you selected in the four previous rounds. You will select one of these four options for this task.

For example, if in the first round you select option $B$ from $A, B, C$, and $D$, in the second round you select Option $F$ from $E, F, G$ and $H$, in the third round you select Option I from Options I, J, K, and L, and in the fourth round you select Option P from Options M, N, O and P, then in the fifth round, you would select among options B, F, I, and P. You select an option by clicking the "select" button corresponding to the option you want. When you press the "select" button, a dialog box will open asking you to confirm your choice.

Please select your option for the task below. We will determine your payment using a deck of cards as explained in the instructions. The deck of cards that applies to this decision is shown below the table of options.

\section{Decision Task - Select One Option}

-Instructions

In this task, you will select one option from 16 possible options: $A, B, C, D, E, F, G, H, I, J, K, L, M, N, O$, and $P$. All 16 options are presented at once. You select an option by clicking the "select" button corresponding to the option you want. When you press the "select" button, a dialog box will open asking you to confirm your choice.

Please select your option for the task below. We will determine your payment using a deck of cards as explained in the instructions. The deck of cards that applies to this decision is shown below the table of options. 


\section{Decision Task - Keep One Option}

-Instructions

In this task, you will select one option from 16 possible options: $A, B, C, D, E, F, G, H, I, J, K, L, M, N, O$, and $P$. This choice is made in a series of five rounds.

In the first round, you will be shown 4 options: A, B, C, and D. You will select one of these four options to keep. In the second round, the three options you did not select will be discarded and three new options (E, F, and G) will be added to the one you kept. Again, you will select one of these four options to keep. This process will continue for three more rounds until you have selected your option for this task and have discarded the other 15 options.

For example, if in the first round you select option $B$ from $A, B, C$, and $D$, then, in the second round, you would see options $B, E, F$, and $G$. If you select Option F in the second round, you would see Options F, H, I, and J in the third round, and so on. You select an option by clicking the "select" button corresponding to the option you want. When you press the "select" button, a dialog box will open asking you to confirm your choice.

Please select your option for the task below. We will determine your payment using a deck of cards as explained in the instructions. The deck of cards that applies to this decision is shown below the table of options. 


\section{Decision Task - You Decide}

Instructions

In this task, you will again select one option from 16 possible options: $A, B, C, D, E, F, G, H, I, J, K, L, M, N, O$, and $P$. However, you will help decide the method that will be used to select an option. You have now gone through three methods for selecting an option:

Send to Final - Four different options are shown in each of the first four rounds. The four options selected in these rounds are shown in the fifth round from which one is selected.

Select One Option - All 16 options are shown at once and a single option is selected.

Keep One Option - Four options are displayed initially. The selected option is kept, while the other three options are replaced with new ones. This process repeats until all 16 options have been displayed and a single option has been selected in the fifth round.

To determine which method will be used for this task, you should answer the following questions which ask you to order the three methods from your most preferred method to your least preferred method.

The computer will randomly eliminate one of the three methods. You will complete the task using your preferred method from the two remaining methods. For example, suppose you indicate that your most preferred method is Send to Final and that your least preferred method is Keep One Option. If the computer eliminates Keep One Option or Select One Option, you would use Send to Final for the fourth task since you indicated that it is your most preferred method. If the computer eliminates Send to Final, then you would use Select One Option for the fourth task as you indicated that you prefer it to Keep One Option. Notice that there is a two-thirds chance you will use the method you indicate you prefer most and a zero chance that you will use the method you prefer least. Therefore, it is in your best interest to answer the questions truthfully, which is why this procedure is used.

When you have finished selecting the three methods and are ready to proceed to the fourth task, press the button below.

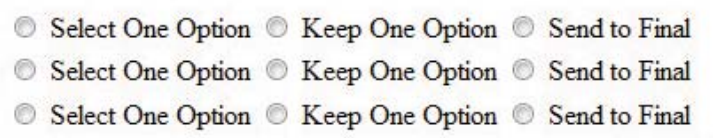

continue 